\title{
A Numeric Application Using Diffusion Limited Aggregation Model for the Manganese Dendrites
}

\author{
Mehmet Bayirli and Hakan Kockar \\ Physics Department, Science and Literature Faculty, Balikesir University, Cagis, 10145, Balikesir, \\ Turkey \\ Reprint requests to M. B.; Fax: +(90) 266 6121215; E-mail: mbayirli@balikesir.edu.tr
}

Z. Naturforsch. 65a, 777 - 780 (2010); received May 18, 2009 / revised October 19, 2009

\begin{abstract}
Black deposits known as manganese dendrites often mark the surfaces of magnesite ore. These have fractal appearance, but the origin and characteristics properties of this morphology and the numerical computation of its geometry have been little studied. The shapes and fractal properties of the manganese dendrites from several different origins were analysed by means of the formation mechanism and Monte Carlo simulation study. These are obtained by the stick probability concepts from the dendrites to compact patterns as the geometrical structures using the diffusion-limited aggregation model. The fractal dimensions were also computed with the stick probability as the numerical equation. The results of the stick probability parameters are compared with the real and arbitrary simulation data and can be helpful to describe the natural and the experimental dendritic patterns.
\end{abstract}

Key words: Diffusion-Limited Aggregation (DLA); Crystal Structure of Minerals; Numerical Methods.

PACS numbers: 61.43.Hv, 91.60.Ed, 02.60-x

\section{Introduction}

In recent years, the pattern formation of nonequilibrium leading naturally to self-similar fractal structures have received great interest not only due to their relevance for various physical process such as electrochemical deposition [1,2], but also because such harmonic growth leads naturally to one of the most interesting multi-fractal distributions found in nature $[3,4]$.

The unstable growth of surface crystallization typically results in abundant geometrical patterns. One of the natural examples of these patterns are manganese dendrites. In palaeontology, dendritic manganese crystal forms are often mistaken for fossils. These, black, fern-like, red-brown natural patterns, normally deposited on the fracture surfaces in rocks or ore, are formed from manganese oxides and iron that has been leached from surrounding rocks and soil by water when supersaturated solutions of iron or manganese penetrate the limestone and are precipitated on exposure to air at the surface [5]. There is a variety of manganese oxides and hydroxides that are birnessite $\left(\mathrm{Na}_{4} \mathrm{Mn}_{14} \mathrm{O}_{27} \cdot 9 \mathrm{H}_{2} \mathrm{O}\right)$, coronadite $\left(\mathrm{PBMn}_{8} \mathrm{O}_{16}\right)$, cryptomelane $\left(\mathrm{KMn}_{8} \mathrm{O}_{16}\right)$, hollandite $\left(\mathrm{BaMn}_{8} \mathrm{O}_{16}\right)$, romanechite $\left(\left(\mathrm{Ba}, \mathrm{H}_{2} \mathrm{O}\right) \mathrm{Mn}_{5} \mathrm{O}_{10}\right)$, and todorokit ((Ba,Mn, $\left.\mathrm{Mg}, \mathrm{Ca}, \mathrm{K}, \mathrm{Na})_{2} \mathrm{Mn}_{3} \mathrm{O}_{12} \cdot 3 \mathrm{H}_{2} \mathrm{O}\right)$. A three-dimensional form of dendrite develops generally in fissures in quartz, forming moss agate [6].

The structure and the growth mechanism of manganese dendrites are discussed both theoretical such as diffusion-limited aggregation (DLA) [7, 8], and experimental investigation. In this model, its mechanism requires a starting point or linear sink of energy to create a positive concentration gradient in the surrounding fluid which must be maintained thought the growth process for manganese dendrites. Chopard et al. [5] has proposed the reaction-diffusion model to describe the formation mechanism of tree-like manganese dendrites. García-Ruiz et al. [6] obtained the tree-like manganese patterns as experimental studies and they presented data supporting the idea that manganese dendrites are the mineral record of flow instabilities [6]. Manganese dendrites are divided in seven different patterns according to the geometrical structures and the values of the fractal dimensions [4]. A limited number of bifurcations appears within the photographs obtained in Figure 1.

A hypothesis was proposed to make a relation between the fractal dimension $D$ and the stick probability parameters (SPP) $t$ by applying a numerical equation for the scaling dendritic DLA patterns. An approach to 


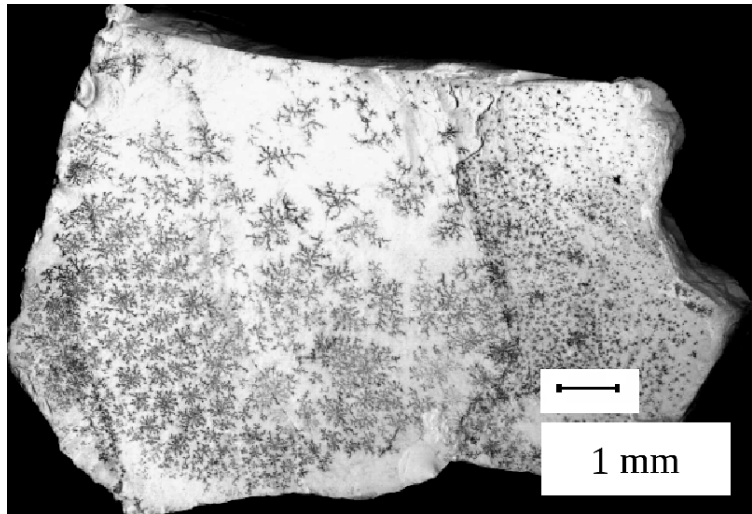

Fig. 1. Images of the natural manganese dendrites formed on the surface of magnetize ore.

the initial parameter estimate functions $D(t)$, using the nonlinear regression method, takes in following form:

$$
D(t)=D_{0}+a \mathrm{e}^{-b t},
$$

where $D_{0}$ is the fractal dimension of DLA, $t$ is the SPP of the aggregations, and $a, b$ are positive constants [7].

These amazing patterns of manganese dendrites have a fractal appearance and natural surface crystallization. To our knowledge, there has been limited information in literature for the numerical origin and characteristics of the morphology and its dependence on the numerical approaches. Because of their very common occurrence, the knowledge of the genetic conditions for manganese dendrites would be of great practical interest in understanding geological environments. Nevertheless, they are considered today to be a rather meaningful structure in the deciphering of geological environments, probability due to uncertainty of their genesis.

In this study, the shapes and fractal properties of the mineral dendrites from several different origins were analysed, and a DLA model for their formation in two dimensions (2D) was proposed. In addition, numerical calculation of $t$ was computed from the real manganese dendrites to use for Monte Carlo (MC) simulation. It is seen that the results can be helpful to describe $t$ for the natural and the experimental dendritic patterns.

\section{Model and Computation}

In this study, the algorithm can be divided into two parts. One of them is the process of producing the standard DLA patterns [8]. The other one is based on the clusters with various patterns that used the stick probability $P$ to obtain each mineral dendrites group using the MC simulation. DLA model can be generalized by introducing the stick probability parameter $t$ of the aggregation. It allows us to vary the fractal dimension $D$ of the crystal patterns. In the generalized DLA model, particles stick to the aggregate on a visiting active site in a square lattice with the stick probability $P$,

$$
P=t^{3-B},
$$

where $t$ is some positive and random adjustable parameter less than $1(0<t \leq 1)$ and $B$ is the number of nearest-neighbour occupied sites in the aggregation [9].

\section{Results and Discussion}

The structure of the manganese dendrites is generally appropriate to $2 \mathrm{D}$-square lattice. In the square lattice, the branches and sub-branches of the dendrites can be grown in all directions. If a triangle lattice was chosen, the brunches can only go towards the corners of the triangle that does not represent the manganese dendrites. Therefore, the MC simulation was performed on a 2D-square lattice system. The length of the square particles was chosen to be unit length. (a)

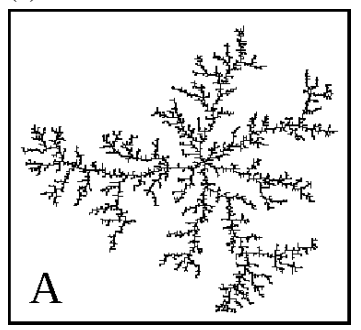

(c)

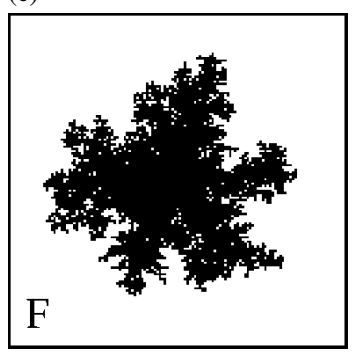

(b)

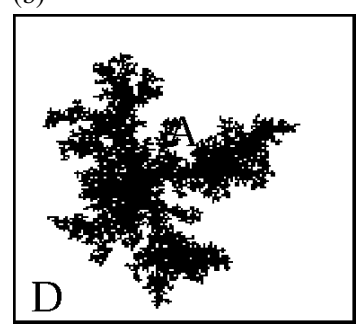

(d)

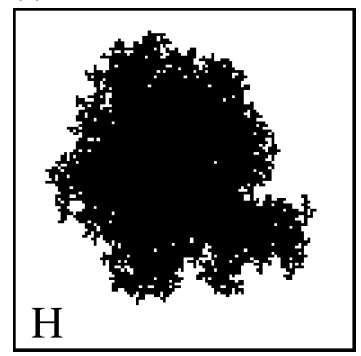

Fig. 2. Morphology of clusters formed by the MC simulation using the generalized DLA ( $L=200, N=20000)$; (a) $t=1$ for group A, (b) $t=0.05$ for group $\mathrm{D}$, (c) $t=0.01$ for group $\mathrm{F}$, (d) $t=0.001$ for group $\mathrm{H}$. 
The SPP was arbitrary taken from 1 to 0.001 to compare the natural manganese dendrites with each other. For each case, seven patterns were performed and eight different clusters were generated for each parameter $t$.

The MC simulation based on the model to create the clusters with various patterns on a square lattice with the different SPP. Figure 2 shows the typical patterns of the DLA clusters growing on the square lattice for different SPP values. As an example, for $t=1$, the DLA cluster appears non-uniform and symmetrical in Figure 2a. Moreover, there are only few branches in this DLA clusters, and the branches have many sub-branches. With the SPP increasing, the number of branches increases and the clusters change to relatively uniform, symmetrical, and compact ones gradually for the rest of the structures in Figure 2. The thickness of the main branches is increased and sub-branches disappear when $t$ decreased in the simulations.

The fractal dimension $D$ of the patterns can reflect the final static property of the surface crystallization. Each manganese dendrite was quantitatively analysed by the digitisation with a resolution of $200 \times 200$ square pixels. The growth zones were approximately found to be confined to the outer $5 \%$ of the patterns. The fractal dimensions were computed by dividing the manganese dendrites into boxes of size $\varepsilon$, and then counting the number of boxes counting occupied pixels as a function of $\varepsilon$, in the usual way for both the simulation patterns and the real manganese dendrites. For the study, although the Hausdorff dimension can be used, which would take longer time, the box-counting method was preferred for the fractal dimensions.

The $D$ values of the particle distributions in the present study can be computed by the box-counting method, which used the random sets in a box [5]. This is defined by covering the fractal with boxes of size $\varepsilon$ and then set

$$
\lambda=\varepsilon / R,
$$

where $R$ is overall of the cluster. Now, the number $N_{\lambda}$ of the boxes can be counted in the covering.

$$
N_{\lambda}=B \lambda^{-D}
$$

where $B$ is a constant and the box-counting dimension $D$ can be obtained from (4).

The first stage of the present study was to confirm the validity of our algorithm. Using the box-counting
Table 1. Arbitrary chosen $t$ values obtained from the MC simulation in the first column. The $t$ values (second column) were computed from $D(t)$ values (third column) from the real manganese images using (5).

\begin{tabular}{llll}
\hline Groups & $\begin{array}{l}\text { Random MC } \\
\text { simulation }(\mathrm{t})\end{array}$ & $\begin{array}{l}\text { Numerical } \\
\text { calculation }(\mathrm{t})\end{array}$ & $\begin{array}{l}\text { Range of } D \\
\text { (from real images) }\end{array}$ \\
\hline $\mathrm{A}$ & $1 \geq t_{\mathrm{A}}>0.4$ & 0.469873 & $(1.524-1.557)$ \\
$\mathrm{B}$ & $0.4 \geq t_{\mathrm{B}}>0.2$ & 0.119971 & $(1.594-1.673)$ \\
$\mathrm{C}$ & $0.2 \geq t_{\mathrm{C}}>0.08$ & 0.090718 & $(1.674-1.680)$ \\
$\mathrm{D}$ & $0.08 \geq t_{\mathrm{D}}>0.06$ & 0.070403 & $(1.686-1.710)$ \\
$\mathrm{E}$ & $0.06 \geq t_{\mathrm{E}}>0.03$ & 0.048491 & $(1.721-1.757)$ \\
$\mathrm{F}$ & $0.03 \geq t_{\mathrm{F}}>0.01$ & 0.014951 & $(1.812-1.846)$ \\
$\mathrm{G}$ & $0.01 \geq t_{\mathrm{G}}>0.0009$ & 0.001746 & $(1.857-1.873)$ \\
$\mathrm{H}$ & $0.0009 \geq t_{\mathrm{H}}>\ldots$ & - & $(1.891-\ldots)$ \\
\hline
\end{tabular}

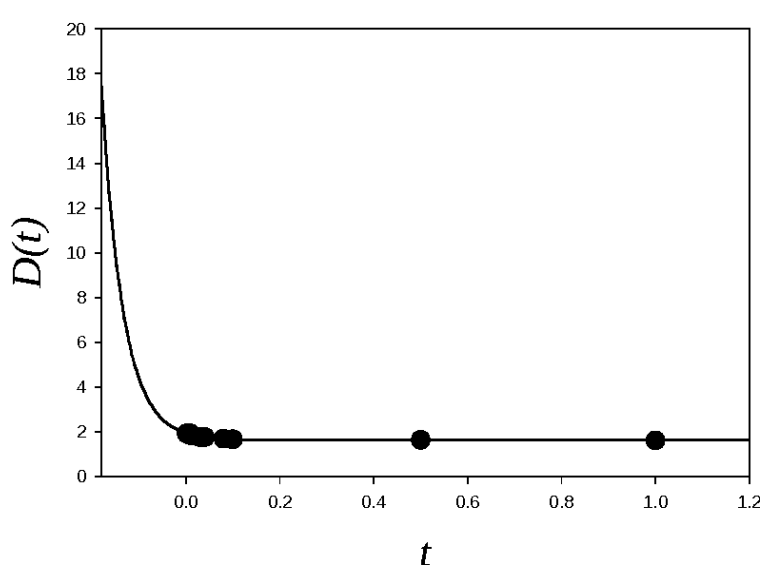

Fig. 3. Plot of the fractal dimension $D$ as a function of $t$ in the range of $1 \geq t>0$ for the MC simulated DLA patterns.

method, the $D$ values of the generated patterns were computed as $D=1.72 \pm 0.01$ for $t=1$. In the standard 2D-DLA patterns, this quantity is close to 1.7 $[8-10]$.

Generated patterns (labelled as A, B, C, and D) obtained by MC simulation show an apparent fractal behaviour between physically relevant cut-offs. The rest of the groups (E, F, G, and $\mathrm{H}$ ) are compact structures. To study the influence of the geometrical structure on the DLA clusters, the functions $D(t)$ of the clusters were plotted in Figure 3. It is found that $t$ decreasing from 1 to 0.001 as $D(t)$ sharply increases from 1.667 to 1.891 . The arbitrary chosen $t$ values for MC simulation are also listed in Table 1.

Now, the numerical relationship between $D(t)$ and $t$ values obtained from the MC simulation can be computed in $2 \mathrm{D}$ space using the nonlinear regression method. If $D_{0}<D(t), D_{0}$ is the fractal dimension for $t=1$, the initial parameters estimate the function of the 
$D(t)$ values and takes in the following form:

$$
t=(1 / b) \ln \left(a /\left(D(t)-D_{0}\right)\right.
$$

where $a$ and $b$ are positive correlation constants. These constants are obtained from the $D(t)$ values by searching for the fit on all regions of the aggregation as reported in [7].

The $D$ values are computed in all real patterns by using the box counting method and are presented in Table 1 . Consequently, the $D$ values were used to numerically calculate the $t$ values using (5). They are also given in Table 1. For group A, the $t$ value obtained from the real image is 0.469873 which is in the range of $1 \geq t>0.4$ of the simulated pattern. It is seen that the rest of the values were also in between the simulated pattern $t$ values.

[1] Y. Sawada, A. Dougherty, and J. P. Gollup, Phys. Rev. Lett. 56, 1260 (1986).

[2] M. Matsushita, M. Sano, Y. Hayakawa, H. Hanjo, and Y. Sawada, Phys. Rev. Lett. 53, 286 (1984).

[3] L. Peterson, Phys. Rev. Lett. 52, 1621 (1984).

[4] M. Bayirli, Physica A 353, 1 (2005).

[5] B. Chopard, H. J. Herrmann, and T. Vicsek, Nature 353, 409 (1991).

[6] J.M. García-Ruiz, F. Otálora, A. Sanchez-Navas, and F. Higes-Rolando, in: Fractals and Dynamics Systems

\section{Conclusion}

In this paper, the generalised DLA model on uniform structure was investigated by means of Monte Carlo simulations in order to study the natural manganese dendrites groups. The SPP values obtained from the DLA model can determine the morphology of the manganese dendrites groups. The numerical equation obtained from $D(t)$ and $t$ values using the MC simulation can be used to only compute the $t$ values of the images of manganese dendrite. It is seen that the numerical approach can be effectively applied on the dendritic structures.

\section{Acknowledgements}

We thank Rifat Capan and Ziya Merdan for contribution and helpful suggestions.

in Geosciences (Ed.: J.H. Kruhl), Springer Verlag, New York 1994, p. 307.

[7] M. Bayirli, J. Optoelectron. Adv. Mater. Symposia 1 3, 547 (2009).

[8] T. A. Witten and L. M. Sender, Phys. Rev. Lett. 47, 1400 (1981).

[9] T. A. Witten and L. M. Sender, Phys. Rev. B 27, 5686 (1983).

[10] T. Vicsek, Fractal Growth Phenomena, Word Scientific, Singapore 1992. 